\title{
Investigating the Role of Wind Turbine Pitch using CFD
}

\author{
Dorit Sobotta ${ }^{1}$, Robert Howell ${ }^{2}$ and Lou Jing ${ }^{3}$ \\ ${ }^{1}$ German Aerospace Centre, Rotorcraft, Institute of Flight Systems, Lilienthalplatz 7, 38108 Brunswick, Germany \\ ${ }^{2}$ Department of Mechanical Engineering, The University of Sheffield, Western Bank, Sheffield, S10 2TN, UK \\ ${ }^{3} A^{*} S T A R$, Institute of High Performance Computing, Fusionopolis, 1 Fusionopolis Way, \#16-16 Connixes, 138632 Singapore
}

\begin{abstract}
Horizontal axis wind turbines are an attractive renewable energy source due to their very low carbon emission during their life cycle. In this study the effect of pitching the rotor blades of the NREL Phase VI rotor has been investigated in detail using CFD in order to allow a detailed torque analysis. Initial investigations have shown that at low rotational speeds the inboard section of the blade is responsible for the majority of the power generation. As the rotational speed increases the power producing section is shifted towards the blade tip. These trends are less pronounced when the blade is pitched which allows the blade to generate significantly more power at low rotational speeds. The improved low speed performance however comes at the cost of a significantly lower maximum power output. These findings are particularly relevant for turbines operating in unfavourable wind environments and for small scale turbines which solely rely on their aerodynamic torque for starting.
\end{abstract}

\section{Introduction}

Wind turbines have experienced a significant increase in their number over the last years as they have a very low carbon footprint of only $4.64 \mathrm{~g}$ of $\mathrm{CO}_{2}$ equivalent per $\mathrm{kWh}$ over their life cycle, most of which occur during their manufacturing and construction [1]. A mechanism common to all of today's large scale rotors is their ability to adjust their blade pitch based on their current wind environment in order to maximise their power output. Several studies, such as those of the WKA-60 rotor [2], have been conducted to investigate the effect of blade pitch. These studies typically only address their torque and power characteristics but not the underlying physical phenomenon, although fully understanding relevant characteristics could aid in the design process of blades that perform well over a wide range of different wind environments. This could not only help designing more efficient large scale rotors but also more efficient small scale rotors as they often operate in unfavourable wind environments and solely rely on their aerodynamic torque for rotor starting.

It is the aim of this study to investigate these shortcomings by analysing the effect of blade pitch which modifies the blade surface inclination with respect to the rotational plane. This in turn influences the pressure-totorque translation and hence the rotor's power output. The modified surface inclination also causes a change in power output by provoking a different pressure field around the blade. This is also reflected in a different near blade flow field. In order to quantify these effects, a Computational Fluid Dynamics (CFD) analysis has been conducted using the geometry of the Phase VI rotor from the National Renewable Energy Laboratory (NREL) as a reference rotor [3]. Due to the availability of extensive experimental data, the two-bladed upwind rotor with a radius of $5.029 \mathrm{~m}$ shown in Figure 1 also served for validating the CFD model.

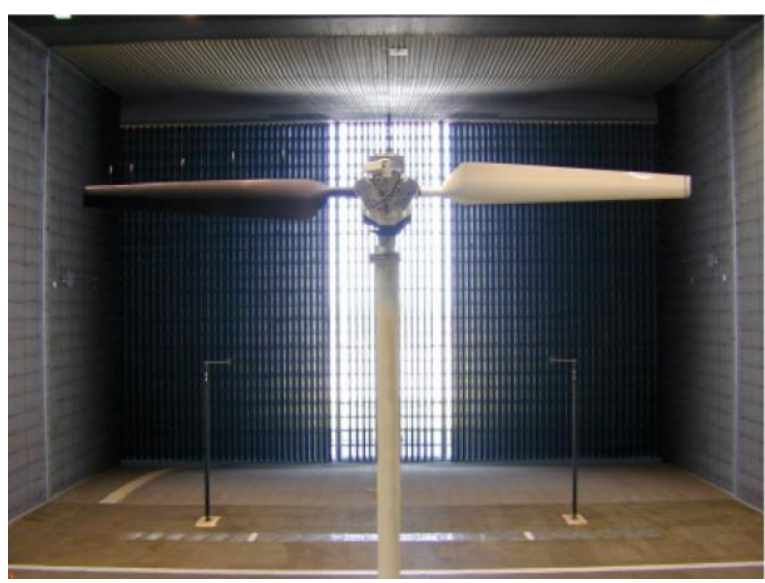

Figure 1. Experimental NREL Phase VI Turbine in NASA's Ames Research Centre [3].

\section{Computational Methods}

In order to investigate the flow around the turbine using CFD, suitable computational methods have to be established first. This section is concerned with the evaluation of suitable turbulence models and meshes which arise due to the closure of the Reynolds Averaged Navier Stokes (RANS) equations and the spatial discretisation of the flow field respectively. In this study 
the CFD solver Ansys Fluent versions 12 [4] and 14 along with the meshing software Pointwise Gridgen version 15 [5] has been used.

\subsection{Turbulence Model Selection}

For computational efficiency only one and two equation turbulence models have been considered for this study. The one equation Spalart-Allmaras (S-A) model and selected models from the two equation $k-\varepsilon$ and $k-\omega$ turbulence model families have been validated against experimental aerofoil data from the NACA $65_{4}-421$ [6]. For the simulation a high resolution $2 \mathrm{D} \mathrm{O}-$ mesh has been used which extends for 20 chord lengths radially outwards. The aerofoil has 320 nodes on its surface which are clustered towards its leading and trailing edge.

The first cell height is such that it satisfies the corresponding turbulence models near wall requirement and extends with a growth rate of 1.06. Table 1 summarises the simulated error of the aerodynamic coefficient. Based on the findings the k- $\omega$ SST model has been chosen for all further investigations due to its comparatively little overall error of the lift, drag and moment coefficient $\mathrm{c}_{\mathrm{L}}, \mathrm{c}_{\mathrm{D}}$ and $\mathrm{c}_{\mathrm{M}}$ respectively. This model has often been used for turbines [7].

Table 1. Percentage Error of Investigated Turbulence Models.

\begin{tabular}{|c|c|c|c|c|c|}
\hline \multirow{2}{*}{} & \multirow{2}{*}{$\mathrm{S}-\mathrm{A}$} & \multicolumn{2}{|c|}{$\mathrm{k}-\varepsilon$} & \multicolumn{2}{c|}{$\mathrm{k}-\omega$} \\
\cline { 3 - 6 } & & Stand. & RNG & Stand. & SST \\
\hline $\mathrm{c}_{\mathrm{L}}$ & -41 & -8 & -5 & -14 & -22 \\
\hline $\mathrm{c}_{\mathrm{D}}$ & +131 & +82 & +41 & +57 & +20 \\
\hline $\mathrm{c}_{\mathrm{M}}$ & -25 & +30 & +33 & +21 & +6 \\
\hline
\end{tabular}

\subsection{Mesh Selection}

After the identification of a suitable turbulence model, a suitable turbine mesh that allows fast, yet accurate computation has been established. A variety of different mesh types including fully structured, hybrid and unstructured have been used in literature. For the current study a hybrid mesh has been adopted which consists of a fully structured high resolution boundary layer mesh, an unstructured transition region and a structured far-field block as outlined in Figure 2. The graph also outlines the domain extent and the boundary conditions.

Table 2. Cell Count x $10^{6}$ of Mesh Depicted in Figure 2.

\begin{tabular}{|l|c|c|}
\hline Mesh & Total & Boundary Layer \\
\hline Very Coarse & 4.2 & 0.6 \\
\hline Coarse & 5.2 & 1.3 \\
\hline Medium & 6.2 & 2.6 \\
\hline Fine & 12.6 & 6.9 \\
\hline
\end{tabular}

The outlined mesh has been used with meshes of 4.2 to 12.6 million cells as displayed in Table 2. While the rotational speed of the two bladed rotor was kept constant at $72 \mathrm{rpm}$, while the wind speed was adjusted to match the blade tip to wind speed ratio $\lambda$, shown in Figure 3 . The resulting distribution of the pressure coefficient $c_{\mathrm{Pr}}$ indicates that the very coarse mesh shows significant mesh dependence at the inboard and outboard blade sections where the flow around the blade is partially stalled at a $\lambda$ of 2.5. At lower and higher $\lambda$ all grids yield mesh independent results which agree reasonably well with experimental data. Consequently the 5.2 million cell mesh has been used for the analysis in this work.

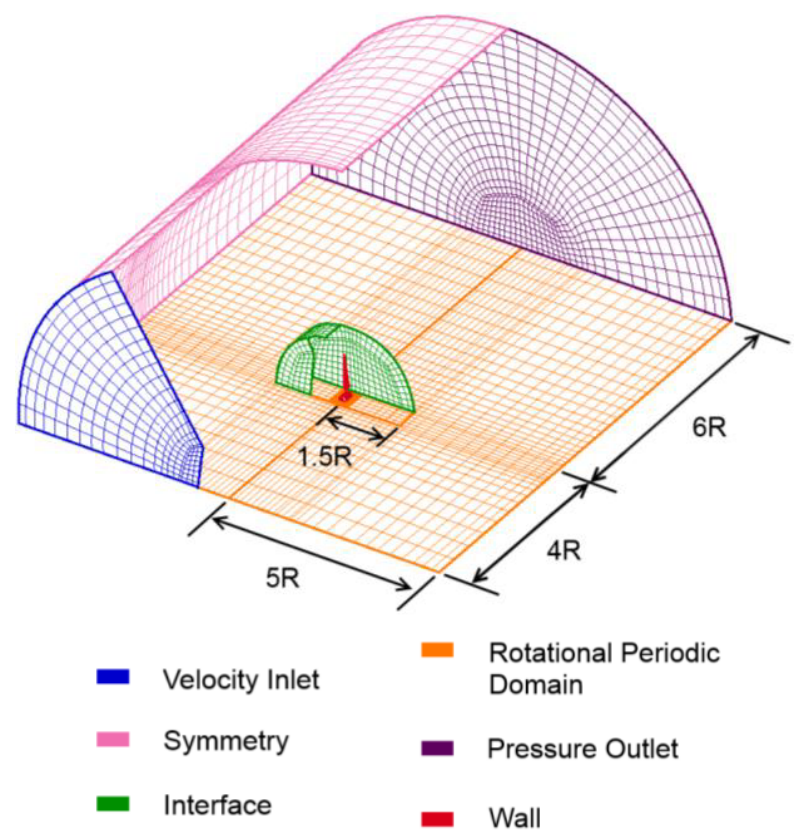

Figure 2. Mesh Topology, Boundary Conditions and Domain Extent.

\section{The Source of Rotor Power}

In order to gain a better understanding of the source of the power produced by a Horizontal Axis Wind Turbine (HAWT), an analysis of its local torque generation characteristics has been conducted in this section. Figure 4 shows the NREL Phase VI turbine's power and torque coefficients, $c_{\mathrm{P}}$ and $\mathrm{c}_{\mathrm{T}}$, as a function of $\lambda$ from a stationary rotor. For this and all following studies the wind speed was kept constant at $6 \mathrm{~m} / \mathrm{s}$ while the rotor speed has been adjusted to simulate $0.5 \lambda$ increments.

From Figure 4 it can be seen that the rotor reaches its maximum $c_{P}$ of 0.29 at a $\lambda$ of 5.6 , while its maximum torque has already been reached at $\lambda=4.5$. In order to further increase its power production beyond this $\lambda$, the decline of its torque production is thus compensated by a higher rotational speed. This already indicates a high sensitivity of the torque the rotor produces towards its highest attainable $c_{P}$. A succeeding study investigates the source of the torque along the blade radius to identify critical blade regions at different $\lambda$. This provides the base to identify the source of differences in rotor power when pitching a blade.

To gain an overview of the radial torque of NREL Phase VI rotor, the spanwise torque distribution has been computed and normalised with the total torque of the respective $\lambda$. The resulting graph is depicted in Figure 5. The normalised torque contour has been overlaid with the local Reynolds number, Re, and the local geometric angle 
of attack, $\alpha$, which is the angle between the local chord line and the resulting velocity vector from the wind speed and rotational velocity. The lines therefore give an indication of the expected local aerodynamic performance; however, their accuracy decreases with increasing $\lambda$ as the induced velocities have not been accounted for. Furthermore, the stripy appearance of the torque contour is due to the underlying mathematical method and does not have any physical meaning.
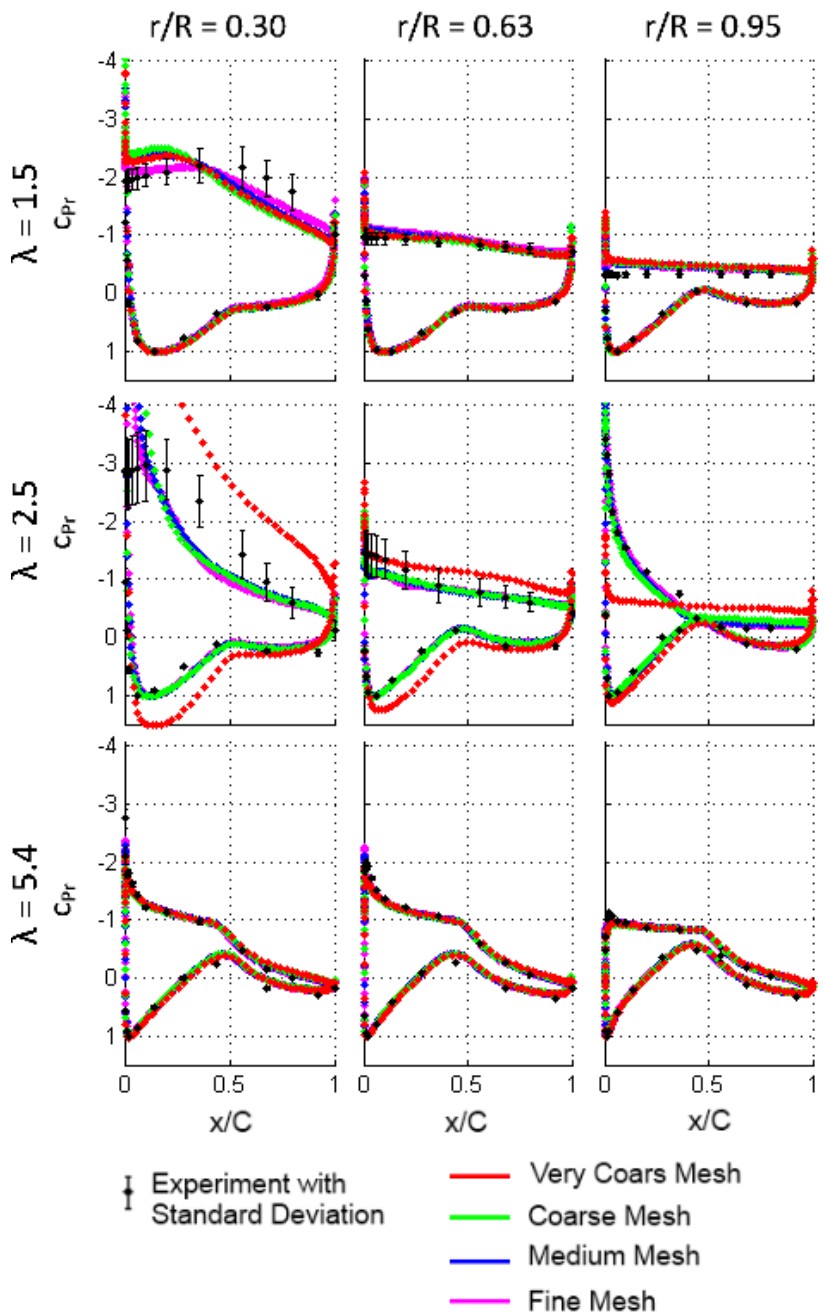

Figure 3. $\mathrm{c}_{\mathrm{Pr}}$ Plots of the Mesh Independence Study of the NREL Phase VI Rotor.

It can be seen that at low $\lambda$ the torque production is predominantly determined by the large blade chord near the blade's root. When Re is nearly uniform along the blade span at $\lambda \approx 2$ the torque production peaks at the blade root. The angle of attack only plays a secondary role. As $\lambda$ increases, the angle of attack at the blade tip drops below that of the blade root. When $\alpha$ further drops below approximately $22^{\circ}$, the torque production at the blade tip begins to overtake that at the blade root and $\mathrm{Re}$ plays a comparatively insignificant role. Based on the current analysis three distinct $\lambda$ sections have been identified:

- $\quad 0.0 \leq \lambda<2.0$ : performance dominated by $\mathrm{Re}$

- $2.0 \leq \lambda<4.3$ : performance dominated by $\alpha$

- $4.3 \leq \lambda \leq 5.5$ : decreasing $\mathrm{c}_{\mathrm{T}}$ with increasing $\lambda$

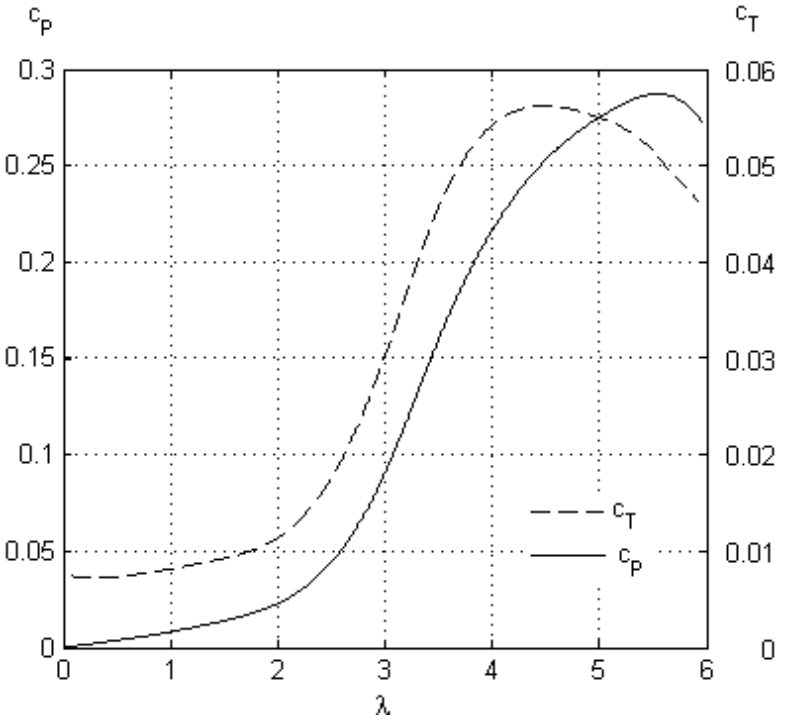

Figure 4. Power and Torque Curve, NREL Phase VI Rotor.
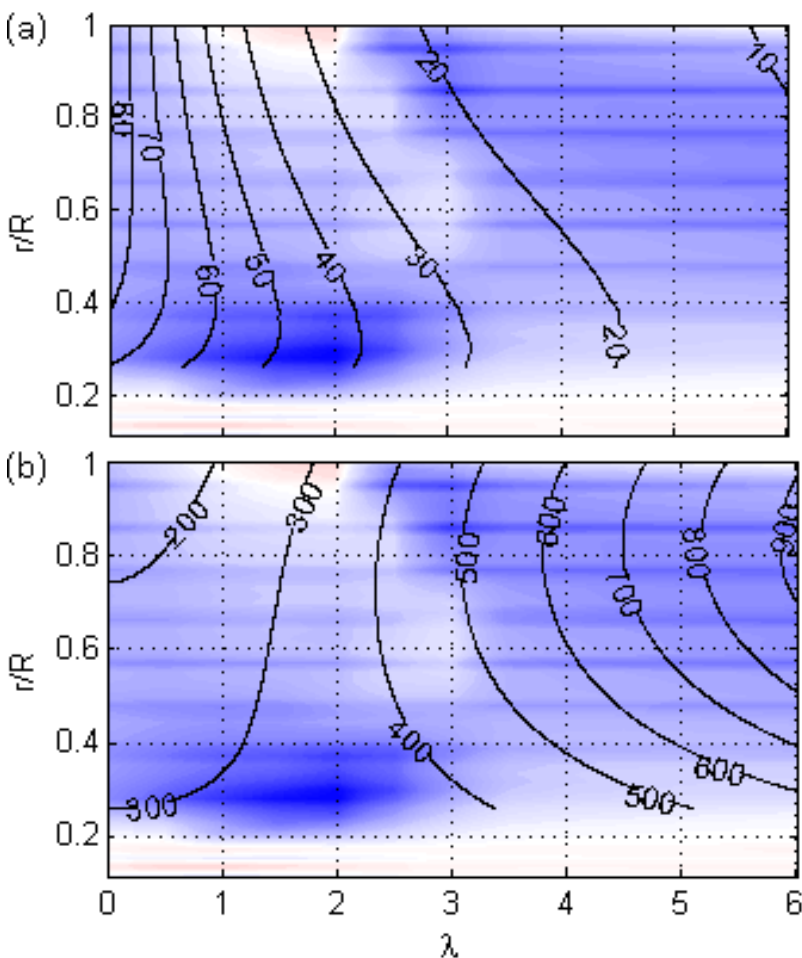

- Max

0

Max

Figure 5. Normalised Radial Torque Distribution of Phase VI Rotor at $0^{\circ}$ Pitch vs. $\lambda$, Superimposed with Geometrical (a) $\alpha$ and (b) $\operatorname{Re} \times 10^{3}$.

When analysing wind turbines, static pressure contours are often presented. These, however, do not allow any direct conclusions about the overall blade performance to be made. In this study the importance of the surface orientation and the blade pitch on translating a given pressure into torque acting in the rotational plane has been highlighted. A given pressure may act to produce useful torque or try to slow the blade down depending on where it acts, for instance near the leading edge or trailing edge of the blade. For demonstration purposes the 
analysis illustrated in Figure 6 has been conducted using a stationary blade as this produces almost exclusively positive and negative static pressure on the pressure and suction surface respectively. Despite each blade surface experiencing no static pressure reversal, the resulting torque distribution shows a change in sign on each surface. This is caused by the orientation of the local blade surface orientation with respect to the rotational plane. The alignment of these two lines is referred to as the zero-inclination-line. When a given static pressure passes this line a change in the direction of the torque produced is thus induced.

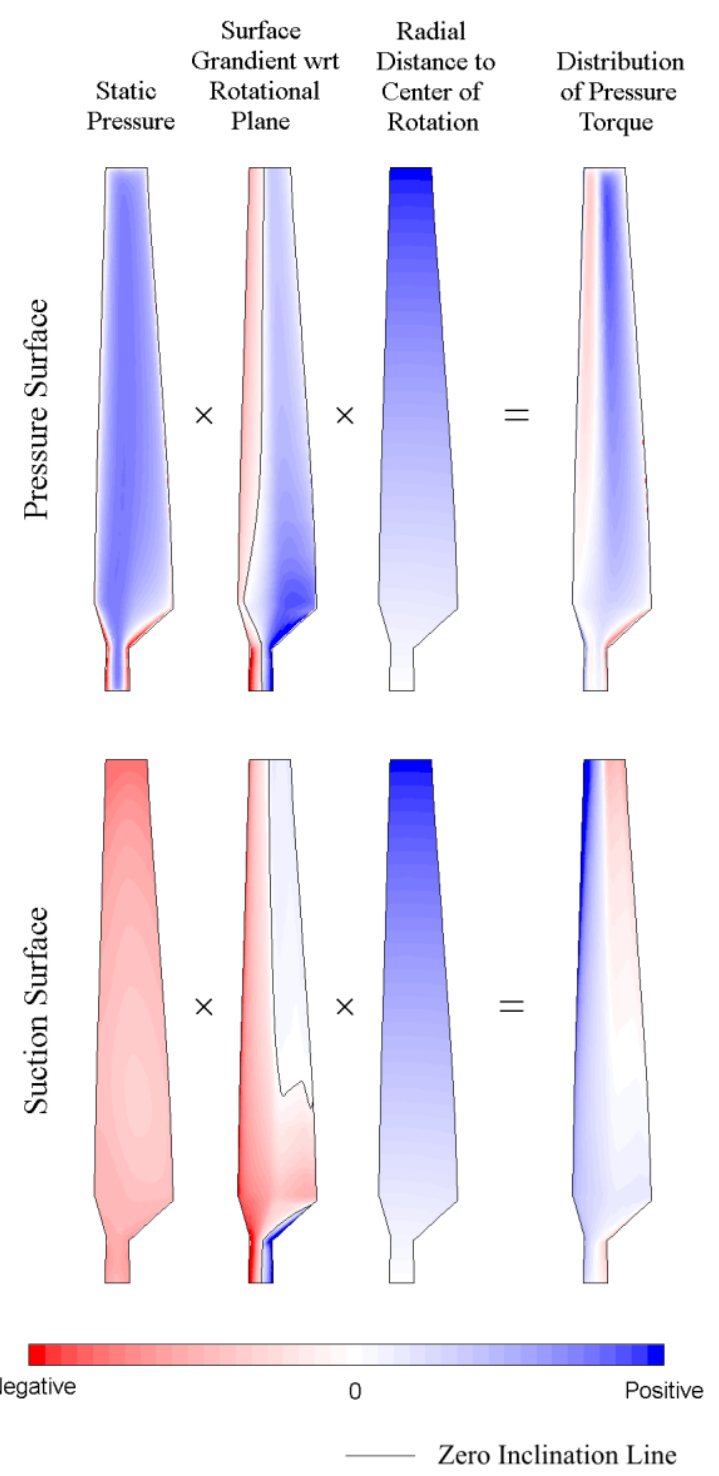

Figure 6. Static Pressure to Pressure Torque Transformation at $\lambda=0.0$.

For a stationary blade it has been observed that the area encompassed between the leading edge and the zeroinclination-line produces positive torque. The remaining section of the blade produces negative torque which acts to slow the blade down. As the pressure surface's torque favouring area outweighs the suction surface's torque retarding area, $63 \%$ of the total torque is produced by the pressure when the blade is stationary.

\section{Effect of Blade Pitch}

After having established relevant criteria influencing the performance of the NREL Phase VI rotor, the blade pitch has been analysed by pitching the NREL Phase VI rotor by $10^{\circ}$ into the wind, thus reducing the angle of attack. This relatively large pitch has been chosen to clearly demonstrate the effect of pitching in 2 sections.

\subsection{Blade Shape}

The first section of the analysis investigates the change of the pressure-to-torque translation properties of the Phase VI rotor when applying pitch. Figure 7 shows the surface orientation of the pitched blade which may be compared to Figure 6. For an easier comparison the line of where the surface orientation of the unpitched rotor is aligned with the rotational plane is also included.

$\begin{array}{ll}\text { Pressure } & \text { Suction } \\ \text { Surface } & \text { Surface }\end{array}$

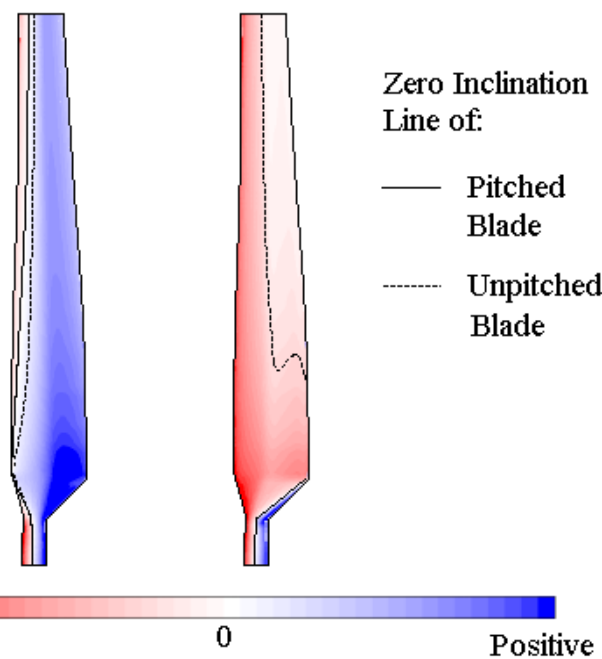

Figure 7. Differences in Blade Surface Orientation of Pitched and Un-Pitched Blade.

Figure 7 indicates that the zero-inclination-line on the suction surface is completely shifted towards the leading edge. Consequently, when the suction surface experiences a negative static pressure, more torque is generated due to a more favourable pressure-to-torque translation. The pressure surface also experiences an increase in its torque production for a given positive pressure distribution as the zero pressure surface line has also been shifted towards the leading edge.

\subsection{Torque Distribution}

The combined effect of the modified blade surface orientation on each blade surface of the rotor torque is illustrated in Figure 8. As expected, the pitched rotor reaches a lower maximum power at a lower $\lambda$ than the reference turbine. Its torque generation at low $\lambda$, however, is superior to that of the unpitched turbine. When the blade is stationary the pitched rotor produces 1.8 times the torque of the unpitched blade. Here the suction surface particularly benefits from the better pressure-to- 
torque translation shown in Figure 7 as it produces 2.2 times the $\mathrm{c}_{\mathrm{T}}$ of the reference blade.

The rapid increase of $c_{T}$ with increasing rotational speed from $\lambda=2.3$ onwards for the Phase VI rotor is now shifted towards 1.4 which allows the turbine to perform well in the low $\lambda$ regime. The following torque peak which occurs at $\lambda=2.8$ is reduced by $22 \%$ when pitching the rotor by $10^{\circ}$. Thereafter, the pitched rotor reaches its optimum performance of only $\mathrm{c}_{\mathrm{T}}=0.127$ at $\lambda=3.2$.

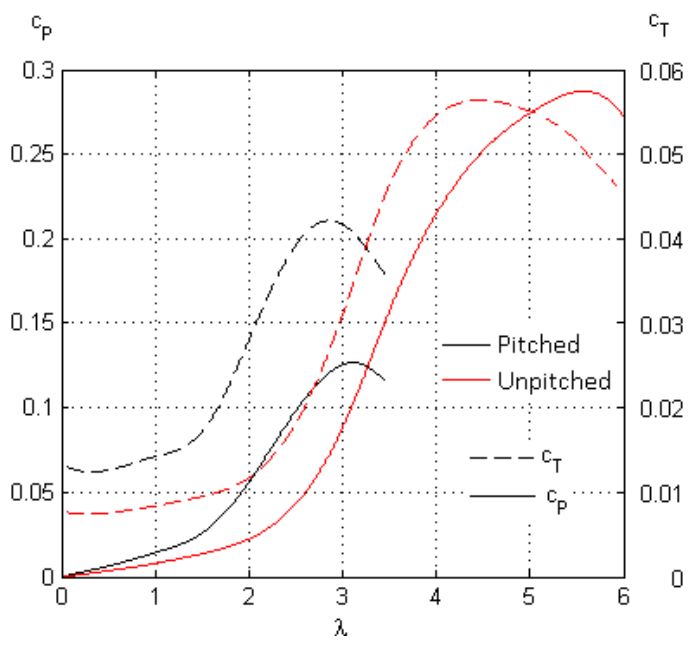

Figure 8. Power and Torque Curve of the Pitched NREL Phase VI Rotor.

A more detailed analysis of the radial dependence of the torque is shown in Figure 9. In contrast to the unpitched blade, the pitched blade shows a very weak Re dependence at low $\lambda$, thus also allowing the outboard section to produce useful torque. The relatively evenly distributed radial torque causes the following increase in the torque production at the blade tip to be much less pronounced than for the unpitched blade. It has also been noted that the pitched blade experiences its $\mathrm{c}_{\mathrm{T}}$ increase only when $\alpha$ drops below approximately $20^{\circ}$. As this occurs at significantly lower $\lambda$, the pitched turbine reaches its optimum rotational speed sooner than the unpitched rotor and thus delivers a lower power output.

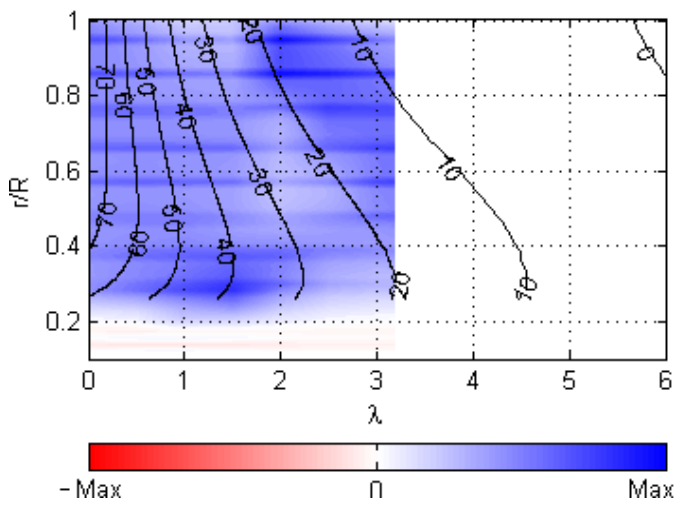

Figure 9. Normalised Radial Torque Distribution of Phase VI Rotor at $10^{\circ}$ Pitch vs. $\lambda$, Superimposed with Geometrical $\alpha$.
The observed trends make the pitch of a blade of a HAWT a suitable mechanism for adjusting to low or unsteady wind environments. Particularly smaller rotors which tend to be placed where they are needed rather than where the wind is best may benefit from pitched blades. When they accelerate from rest they solely rely on their aerodynamic torque as they are usually not fitted with a motor to assist turbine starting for economic reasons.

\section{Conclusions}

It has been identified that at very low $\lambda$ the rotor power is dominated by the local Reynolds number and thus its local chord length. This effect has been observed to diminish when the blade is pitched. As $\lambda$ increases above 2 the resulting torque rapidly increases as $\alpha$ drops below $22^{\circ}$. For the unpitched blade the increase in torque continues until $\lambda=4.3$. Thereafter the gain in the power output is solely due to an increase in rotational speed.

Differences in the torque produced when pitching a blade have been attributed to different pressure-to-torque translation characteristics which are determined by the blade's local surface orientation. The effect of the surface orientation, however, is twofold as it also affects the static pressure distribution around the blade. Pitching a blade causes it to reach its optimum local angle of attack sooner and thus prohibits the blade from reaching high rotational speeds. This thereby reduces a pitched blade's maximum power output. Before reaching its maximum power however, the pitched blade consistently delivers a higher power output than the unpitched blade which makes it suitable for low wind speed environments.

\section{References}

1. N.N., "Carbon Footprint of Electricity Generation", Parliamentary Office of Science and Technology, Number 268, London, UK, 2006.

2. E. Hau, Windkraftanlagen - Grundlagen, Technik, Einsatz, Wirtschaftlichkeit, 4th edition: Springer, Berlin Heidelberg, Germany, 2008.

3. M.M. Hand, et al., "Unsteady Aerodynamics Experiment Phase VI: Wind Tunnel Test Configurations and Available Data Campaigns", NREL/TP-500-29955, NREL, Colorado, 2001.

4. N.N., User's Guide, Ansys Fluent 12.0, Canonsburg, Pennsylvania, 2009.

5. N.N., Version 15 User Manual, Pointwise Gridgen, Forth Worth, Texas, 2006.

6. P. Devinant, et al., "Experimental study of windturbine airfoil aerodynamics in high turbulence", Journal of Wind Engineering and Industrial Aerodynamics, vol. 90, pp. 689-707, 2002.

7. Y.M. Park, et al., "Numerical simulation of wind turbine scale effects by using CFD", in: 45th AIAA Aerospace Sciences Meeting and Exhibit, Reno, Nevada, 2007, pp. 2547-2556. 